\title{
Retrieving permittivity model parameters for polar liquids and multilayer systems through THz-TDS time-trace data analysis
}

\author{
Melanie Lavancier ${ }^{1}$, Sergey Mitryukovskiy ${ }^{1}$, Nabil Vindas ${ }^{1}$, Jean-Francois Lampin ${ }^{1}$, Romain Peretti ${ }^{1}$ \\ ${ }^{1}$ Institut d'Electronique, de Microélectronique et Nanotechnologie (IEMN), CNRS, \\ Université de Lille, Villeneuve d'Asq, France
}

\begin{abstract}
We present new features of the Fit@TDS software for the analysis of polar liquids and multilayer systems. We also show that the modeling of the absorption coefficient of polar liquids can be improved around 2 THz. Hence, this software will enable us to retrieve the parameters of the models depicting polar liquids. It will help to analyze and compare charges motions, playing an important role in biology and chemistry with such effects as protein solvation. Finally, the implementation of a scattering model will improve the accuracy of the results.
\end{abstract}

\section{INTRODUCTION}

$\mathrm{T}$ erahertz time-domain spectroscopy (THz-TDS) is a powerful tool for the characterization of materials. Their parameters, such as the refractive index and the absorption coefficient, are usually retrieved using the Fouriertransform of the recorded time-traces with and without a sample. The ratio between these two time-traces is called the complex transmission coefficient written as :

$\tilde{T}(\omega)=\frac{\widetilde{E}_{s}(\omega)}{\widetilde{E}_{r e f}(\omega)}=\tilde{s}(\omega) \times \exp \left(-j \frac{\omega d}{c}(\tilde{n}(\omega)-1)\right) \times \widetilde{F P}(\omega)$

Where $s(\omega)$ is the product of the Fresnel coefficients at normal incidence for the two air/sample interfaces, the second term corresponds to the propagation into the sample and $F P(\omega)$ is the Fabry-Perot term taking into account multiple reflections in the sample [2].

The Fit@TDS software enables a user to compare the recorded temporal pulse with the modeled one using a robust and generic optimization approach described in $[2,3,5]$. The first version of our software has used a Drude-Lorentz model for solid-state samples and time-domain coupled modes theory for metamaterials:

$$
\tilde{\varepsilon}(\omega)=\tilde{n}^{2}(\omega)=\varepsilon_{\infty}+\frac{\omega_{p}^{2}}{\omega^{2}+j \omega \gamma_{p}}+\sum_{k=1}^{k_{\max }} \frac{\Delta \varepsilon_{k} \omega_{0, k}^{2}}{\omega_{0, k}^{2}-\omega^{2}+j \omega \gamma_{k}}
$$

This allowed the characterization of free charges in metals, oscillating charges in dielectrics and resonances in metamaterials. To extend further the use of the method, we added the Debye model [6] for polar liquids that describes a relaxation feature and a scattering term to separate absorption losses from scattering losses. It is of utmost importance to implement the model for liquids since the intricate molecular processes are linked to the permittivity of the sample, which is more complex to model for them. Indeed, the parameters of the models are not independent because there are two types of dynamics in liquids: free rotation and translation of molecules and interactions between molecules and these interactions cannot be treated separately. Taking into account scattering is as well important in various biological studies since biosamples are often inhomogeneous (emulsions, pellets ...). Both need to be implemented in order to be closer to real samples.

In addition to these models, our software is now able to treat the $\mathrm{THz}$ time-traces from a structure composed of three parallel layers of different dielectric properties. This means the capacity to analyze the sample in its container as a cuvette or a microfluidic cell. This will be consequently useful for samples with several layers especially in the semiconductor field.

\section{SimUlations AND MOTIVATIONS}

Liquid water is of utter importance for biological samples and is still a subject of research in many fields of science. Its complexity relies on several factors, among them the role played by the $\mathrm{H}$-bond is one of the most important. We modeled water in the $\mathrm{THz}$ range with three different models. First, using only one Debye term, then two Debye terms and finally two Debye terms and one Lorentz as we found in the literature they were the most commonly used. To understand the differences adding a term bring, we simulated the transmission, absorption coefficient and the refractive index of these three models. This brought us to the conclusion that the $\mathrm{THz}$ range was adequate to investigate molecular motions as the shapes of the curves were highly impacted by the presence or absence of a term.

The $\mathrm{THz}$ pulse time-trace data was recorded using a commercial THz-TDS experimental setup (TeraSmart by Menlo [4]) purged with nitrogen, allowing spectral measurements between 0.1 and $4 \mathrm{THz}$. The liquid water was contained in a microfluidic cell, built from two z-cut quartz windows separated by a $100-\mu \mathrm{m}$-thick PTFE spacer. The reference pulse time-trace was measured without the microfluidic cell. In Figure 1, the time trace of the data is displayed with the fit from the three models. In Figure 2, the experimentally recovered losses spectrum of liquid water is presented along with the modeled ones for the comparison.

The "1 Debye" model drifts apart from the data around $1 \mathrm{THz}$, which is in accordance with the theory [1] explaining that the relaxation feature happens before $1 \mathrm{THz}$, meaning that at least a second term is needed to represent all the microscopic processes. The second Debye term, a second relaxation feature, indeed brings the model curve closer to the data but the added Lorentz term does not seem to fit the data better. Hence, the vibration represented by this term must occur at higher frequencies and does not appear in our experiment. Moreover, we can see that all models fail to represent the data around $2 \mathrm{THz}$, which has to be investigated to find out which molecular process triggers this change. 

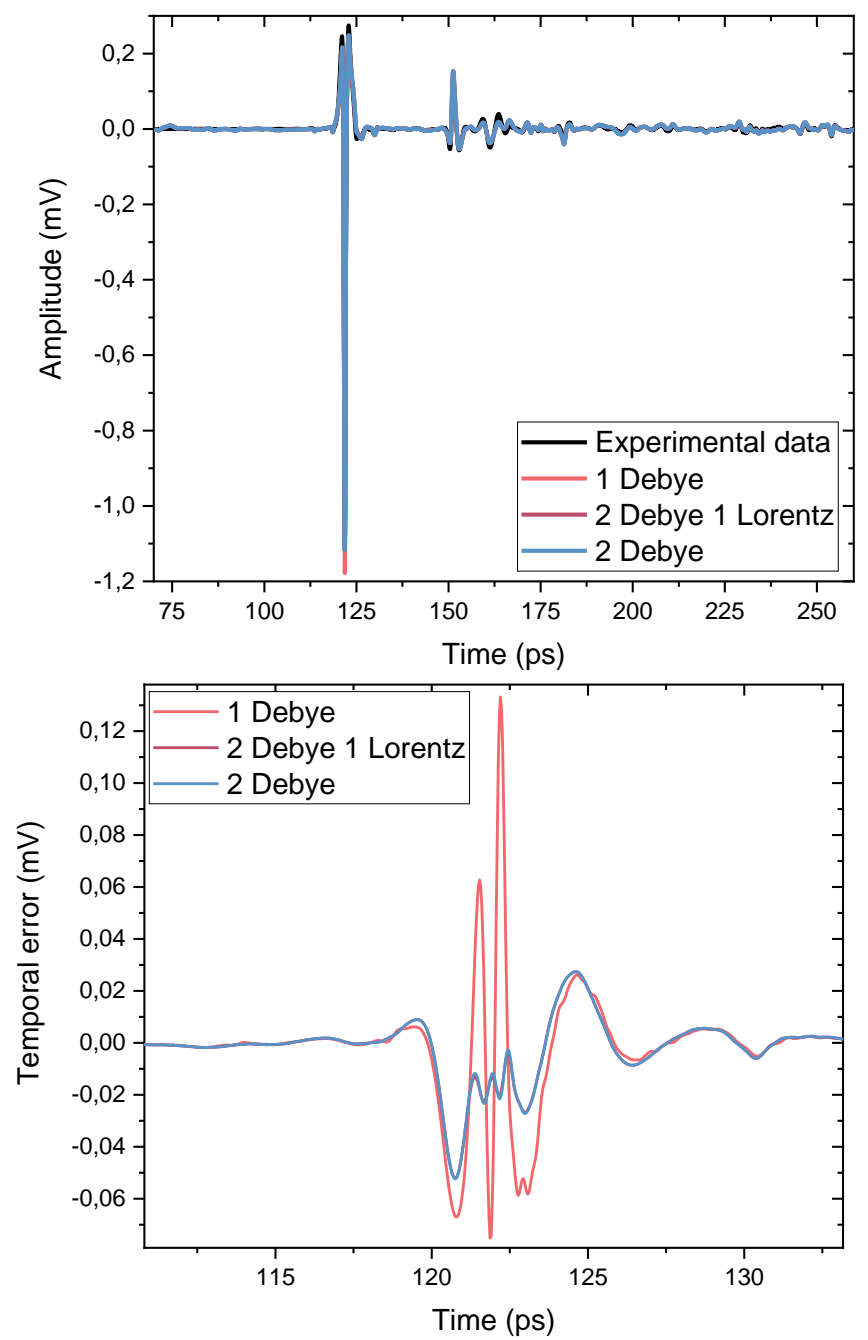

Fig. 1. (a) Time-trace of the experimental data (in black) with the result of the temporal fit with three models (1 Debye term - pink, 2 Debye terms blue, 2 Debye terms and 1 Lorentz term - purple). (b) Difference between the experimental pulse and the fitted pulse for the three models, zoomed on the first pulse.

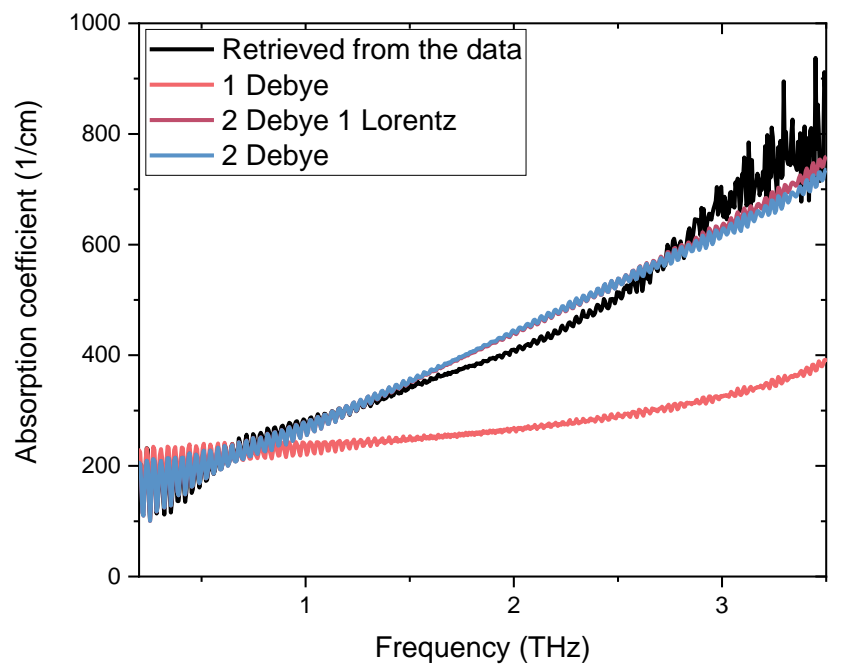

Fig. 2. Spectrum of the absorption coefficient of liquid water calculated using the three models (1 Debye term - pink line, 2 Debye terms - blue, 2 Debye terms and 1 Lorentz term - purple) and the experimental data (in black).

\section{SUMMARY}

In this work, we have presented the new features of the Fit@TDS software that allows the fit of the THz-TDS data with material and metamaterial models based on a timedomain fit. Solid-state samples, metals and metamaterials could already be investigated but new kinds of samples can now be fitted: polar liquids, multilayer samples and scattering samples. This has been applied on liquid water contained in a microfluidic cell allowing us to raise questions on the microscopic processes occurring around $2 \mathrm{THz}$.

In the future, we will go a step further and use the Fit@TDS software to fit experimental data of other polar liquids, including but not limited to water, in order to extract the relaxation parameters and to investigate if there are similar features in the fits. This will allow us to get a better understanding of molecular motions in liquid samples, to compare different polar liquids and to detect the effect of the additional components such as enzymes. These will then be combined with the scattering model to discriminate the different phases of an emulsion and to follow motion parameters in such solutions.

\section{ACKNOWLEDGMENTS}

This work was supported in part by the International Chair of Excellence "ThOTroV" from region "Hauts-de-France", in part by the Welcome Talent Grant "NeFiStoV" from the European Metropole of Lille, in part by the "TeraStoVe" from I-site ULNE, and in part by the French government through the National Research Agency (ANR) under program PIA EQUIPEX ExCELSiOR ANR 11-EQPX-0015.

\section{REFERENCES}

[1]. O.A. Smolyanskaya, et al, "Terahertz biophotonics as a tool for studies of dielectric and spectral properties of biological tissues and liquids", Progress in Quantum Electronics 62, 1-77, 2018.

[2]. R. Peretti, et al, "THz-TDS time-trace analysis for the extraction of material and metamaterial parameters", IEEE transactions on Terahertz Science and Technology, 9, 136, 2019. Software available on: https://github.com/THzbiophotonics/Fit-TDS.

[3] R. Peretti, et al, "Retrieving material and metamaterial parameters directly from time-domain spectroscopy time trace", 43 rd International Conference on Infrared, Millimeter, and Terahertz Waves (IRMMW-THz), Nagoya, 2018.

[4]"http://www.menlosystems.com/en/products/thz-time-domain-

solutions/terasmart-terahertz-spectrometer/." [Online]. http://www.menlosystems.com/en/products/thz-time-domain-

Available: solutions/terasmart-terahertz-spectrometer/

[5] R. Peretti, et al, "Modeling and parameters retrieving in time-domain spectroscopy of material and metamaterial", SPIE Photonics Europe, Strasbourg, 2018

[6] P. Debye (1913), Ver. Deut. Phys. Gesell. 15, 777; reprinted 1954 in collected papers of Peter J.W. Debye. Interscience, New York 